\title{
A Model for Urban Road Network Vulnerability Analysis Based on Cumulative Prospect Theory
}

\author{
LÜ Biao ${ }^{1,2, a}$, Huang $Y u^{3, b}$ \\ ${ }^{1}$ Department of Computer and Communication Engineering, Emei Branch of Southwest Jiaotong \\ University, Emeishan 614202, China; \\ ${ }^{2}$ Department of Traffic and Transportation, Emei Branch of Southwest Jiaotong University, \\ Emeishan 614202, China; \\ ${ }^{3}$ Department of Transportation and Logistics, Southwest Jiaotong University, Chengdu 610031 , \\ China) \\ ${ }^{a}$ swjtu_lb@126.com b HuangY@my.swjtu.edu.cn
}

Keywords: traffic engineering; road network vulnerability evaluation; cumulative prospect theory; variational inequality; key link identifications; stochastic user equilibrium; travel time budget

Abstract: A model for network vulnerability evaluation based on cumulative prospect theory is proposed for more accurate evaluation of the effects of travelers' behavioral responses on road network vulnerability caused by link closure. In the proposed model, the effects of both demand variations and travelers' route choice behavior with bounded rationality are explicitly taken into account. The calculation formulas of the prospect values are derived when traffic demand follows a log-normal distribution, and the stochastic user equilibrium model based on cumulative prospect theory formulated as an equivalent variational inequality problem is built. A network validity index based on cumulative prospect value is proposed, and the change of network validity is used to evaluate network vulnerability and then identify the critical links that the failure of those links would have significant impacts on the whole network. The results show that both demand fluctuation and travelers' route choice behaviors would have significant effect on consequences of road network vulnerability evaluation. Different demand level, demand fluctuation degree and assumption on travelers' route choice behaviors would lead to different results of network vulnerability evaluation.

\section{Introduction}

Urban road network is a complex giant system with the interaction of human, vehicle, road and environment, which is affected by random events, such as natural disasters, man-made attacks and traffic accidents, the decline of capacity may cause the traffic jams and even lead to functional failure. The road network vulnerability is defined as the property of decreasing in the network performance or service level due to the loss of connected capacity, whether full or partial network, resulting from the influence of random event ${ }^{[1]}$. Evaluation of road network could provide the basis for the traffic management department to determine the vulnerability degree of link and take further measure, which benefits to enhance the ability in preventing and responding to disaster events.

Current researches on road network vulnerability are still in the initial stage, evaluation methods need to be improved and it has not been a generally accepted evaluation system. Berdica gave the definition of road network vulnerability firstly ${ }^{[2]}$, which was seen as a susceptibility to incidents that can result in considerable reductions in road network serviceability. Jenelius et al ${ }^{[3]}$ calculated the road network vulnerability from the perspective of "equal opportunity" and "social benefits" in northern Sweden. Taylor et al ${ }^{[4]}$ studied the consequences of the degradation of the road network in Australia through three indexes that include the increasing of the generalized travel cost, 
relative decreasing of accessibility of Hessian matrix and the increasing of the distance. Chen et al took the variety between traffic demand and supply into account, and used the accessibility index based on utility to evaluate the road network vulnerability ${ }^{[5]}$. Yang L.P. et al assessed the road network vulnerability according to ultimate loss of travel time ${ }^{[6]}$, and the road network structure, operation state of traffic flow, the setting of emergency rescue point and so on were comprehensively considered.

In view of the above, the research about vulnerability analysis of road network mainly focus on the qualitative assessment method, and the existing quantitative assessment model needs a further improvement for the reason that the change of supply and demand condition and travelers' behavior responses to the interference events are often neglected. Studies have indicated that if travelers' behavior are ignored in the road network vulnerability analysis, the identification of critical links and the judgment of link failure may be wrong, which would result in sub optimal or even incorrect evaluation scheme ${ }^{[7]}$. In addition, there are some psychological and behavioral science studies have revealed that travelers' route choice behavior would present limited rational characteristic under the influence of random events, which is in the framework of cumulative prospect theory (cumulative prospect theory, CPT ${ }^{[8,9]}$.

This study proposes a model for network vulnerability evaluation based on cumulative prospect theory and derive the travel time distribution function under the condition of fluctuating demand. A definition of effective index of road network is given based on the proposed model, then the critical links is identified via the vulnerability of road network that is measured by the vibration of effective index.

\section{Stochastic user equilibrium model based on CPT}

Traveling time under the random demand. Consider a road network represented by a connected graph $G=(N, A)$, where $N$ and $A$ are the set of nodes and links respectively. $W$ denotes the set of origin-destination (OD), $R_{w}$ represents the set of paths between the OD pair $w, w$ belongs to $W$.

It is assumed that OD demand is an independent random variable represented by $Q_{w}$ and follows some statistical distribution. According to the relationship between the OD demand, path flow and link flow, it could be expressed as:

$$
\begin{aligned}
& Q_{w}=\sum_{k \in R_{w}} F_{k}^{w}, w \in W \\
& F_{k}^{w} \geq 0, k \in R_{w}, w \in W \\
& V_{a}=\sum_{w \in W} \sum_{k \in R_{w}} F_{k}^{w} \delta_{a, k}^{w}, a \in A
\end{aligned}
$$

Where $F_{k}^{w}$ and $V_{a}$ respectively are the mean of traffic flow on path $k$ between OD pair $w$ and traffic flow on link $a$. And $\delta_{a, k}^{w}$ is the link-path incidence variable, $\delta_{a, k}^{w}=1$ if path $k$ uses link $a$, otherwise $\delta_{a, k}^{w}=0$.

$q_{w}, f_{k}^{w}$ and $v_{a}$ represent the mean of $Q_{w}, F_{k}^{w}$ and $V_{a}$ respectively, then they can be expressed as :

$$
\begin{aligned}
& q_{w}=E\left(Q_{w}\right)=\sum_{k \in R_{w}} f_{k}^{w}, w \in W \\
& f_{k}^{w}=E\left(F_{k}^{w}\right) \geq 0, \quad k \in R_{w}, w \in W \\
& v_{a}=E\left(V_{a}\right)=\sum_{w \in W} \sum_{k \in R_{w}} f_{k}^{w} \delta_{a, k}^{w}, a \in A
\end{aligned}
$$


Where $E(\cdot)$ is expected value operator.

There are three assumptions for convenient calculation that are same with the similar problems in existing researches ${ }^{[10]}$, (1): the path flow obeys the same probability distribution with the OD demand. (2): the variance-to-mean rate(VMR) of path flow is identical to the OD demand, (3): the path flow is mutually independent.

The variance of $Q_{w}$ and $F_{k}^{w}$ are denoted as $\varepsilon_{Q_{w}}$ and $\varepsilon_{F_{k}^{w}}$ respectively, which could be calculated according to the first two assumptions.

$$
\varepsilon_{F_{k}^{w}}=\operatorname{var}\left(F_{k}^{w}\right)=f_{k}^{w}\left(\frac{\varepsilon_{Q_{w}}}{q_{w}}\right)=f_{k}^{w} V M R_{w}, \quad k \in R_{w}, w \in W
$$

Where $\operatorname{var}(\cdot)$ denotes the variance operator; $V M R_{w}=\varepsilon_{Q_{w}} / q_{w}$ is the $V M R$ between the OD pair $w$.

The variance of path flow is denoted as $\varepsilon_{V_{a}}$, according to the formula 3, formula 7 and the third assumption, calculate $\varepsilon_{V_{a}}$ by

$$
\varepsilon_{V_{a}}=\operatorname{var}\left(V_{a}\right)=\operatorname{var}\left(\sum_{w \in W} \sum_{k \in R_{w}} F_{k}^{w} \delta_{a, k}^{w}\right)=\sum_{w \in W} \sum_{k \in R_{w}} \delta_{a, k}^{w} \varepsilon_{F_{k}^{w}}, \quad a \in A
$$

The normal distribution is always used to describe the random fluctuations of the OD demand in the majority of existing research ${ }^{[11]}$. In this study, log-normal distribution instead of normal distribution is used to describe the random fluctuation of OD demand of which the value may be negative. Assuming that OD demands $Q_{w}$ obeys the log-normal distribution with the mean of $\mu_{Q_{w}}$ and the standard deviation of $\sigma_{Q_{w}}$, namely $Q_{w} \sim L N\left(\mu_{Q_{w}}, \sigma_{Q_{w}}\right), w \in W$. Then the parameters of distribution of $Q_{w}$ can be calculated.

$$
\begin{aligned}
& \mu_{Q_{w}}=\ln \left(q_{w}\right)-0.5 \ln \left(1+\varepsilon_{Q_{w}} /\left(q_{w}\right)^{2}\right), w \in W \\
& \sigma_{Q_{w}}=\sqrt{\ln \left(1+\varepsilon_{Q_{w}} /\left(q_{w}\right)^{2}\right)}, w \in W
\end{aligned}
$$

According to the previous assumption 1 (A1), the path flow $F_{k}^{w}$ has same distribution with the $Q_{w}$ on the condition that OD demands obeys the log-normal distribution. The parameters of LGD of $F_{k}^{w}$ are denotes as $\mu_{F_{k}^{w}}$ and $\sigma_{F_{k}^{w}}$ respectively, that is

$$
\begin{aligned}
& \mu_{F_{k}^{w}}=\ln \left(f_{k}^{w}\right)-0.5 \ln \left(1+\frac{\varepsilon_{F_{k}^{w}}}{\left(f_{k}^{w}\right)^{2}}\right), k \in R_{w}, w \in W \\
& \sigma_{F_{k}^{w}}=\sqrt{\ln \left(1+\varepsilon_{F_{k}^{w}} /\left(f_{k}^{w}\right)^{2}\right)}, k \in R_{w}, w \in W
\end{aligned}
$$

As the path flow obeys the lognormal distribution and the link flow is the result of the superposition of path flow, the link flow approximately obeys the lognormal distribution according to the property that the sum of random variables that obeys the lognormal distribution also obeys the lognormal distribution in the research. $\mu_{V_{a}}$ and $\sigma_{V_{a}}$ are used to represent the parameters of link flow $V_{a}$ obeying the lognormal distribution.

$$
\begin{aligned}
& \mu_{V_{a}}=\ln \left(v_{a}\right)-0.5 \ln \left(1+\varepsilon_{V_{a}} /\left(v_{a}\right)^{2}\right), a \in A \\
& \sigma_{V_{a}}=\sqrt{\ln \left(1+\varepsilon_{V_{a}} /\left(v_{a}\right)^{2}\right)}, a \in A
\end{aligned}
$$

It is assumed that the link travel time $\mathrm{T}_{\mathrm{a}}$ follows widely used Bureau of Public Road (BPR) link performance function:

$$
T_{a}=T_{a}\left(V_{a}, c_{a}\right)=t_{a 0}\left(1+\beta\left(\frac{V_{a}}{c_{a}}\right)^{n}\right), a \in A
$$


Where $t_{a 0}$ is the free-flow travel time on link a; $c_{a}$ is the link capacity; $\beta$ and $n$ are parameters. The mean and variance of the link travel time $T_{a}$ are denoted as $t_{a}$ and $\varepsilon_{T_{a}}$ respectively, as shown below:

$$
\begin{aligned}
& t_{a}=E\left(T_{a}\right)=t_{a 0}+\left(\beta t_{a 0} /\left(c_{a}\right)^{n}\right) E\left(\left(V_{a}\right)^{n}\right), a \in A \\
& \varepsilon_{T_{a}}=\operatorname{var}\left(T_{a}\right)=E\left(\left(T_{a}\right)^{2}\right)-\left(E\left(T_{a}\right)\right)^{2}, a \in A \\
& E\left(\left(T_{a}\right)^{2}\right)=\left(t_{a 0}\right)^{2}+\frac{2 \beta\left(t_{a 0}\right)^{2} E\left(\left(V_{a}\right)^{n}\right)}{\left(c_{a}\right)^{n}}+\frac{\left(\beta t_{a 0}\right)^{2} E\left(\left(V_{a}\right)^{2 n}\right)}{\left(c_{a}\right)^{2 n}}, a \in A
\end{aligned}
$$

As the link flow $V_{a}$ follows log-normal distribution, $E\left(\left(V_{a}\right)^{n}\right)$ and $E\left(\left(V_{a}\right)^{2 n}\right)$ can be calculated to get the value of $t_{a}$ and $\varepsilon_{T_{a}}$.

$$
\begin{aligned}
& E\left(\left(V_{a}\right)^{n}\right)=\exp \left(n \mu_{V_{a}}+\left(n \sigma_{V_{a}}\right)^{2} / 2\right), a \in A \\
& E\left(\left(V_{a}\right)^{2 n}\right)=\exp \left(2 n \mu_{V_{a}}+\left(2 n \sigma_{V_{a}}\right)^{2} / 2\right), a \in A
\end{aligned}
$$

Then

$$
\begin{aligned}
& t_{a}=t_{a 0}+\frac{\beta t_{a 0}}{\left(c_{a}\right)^{n}} \exp \left(n \mu_{V_{a}}+\frac{\left(n \sigma_{V_{a}}\right)^{2}}{2}\right), a \in A \\
& \varepsilon_{T_{a}}=\frac{\left(\beta t_{a 0}\right)^{2}}{\left(c_{a}\right)^{2 n}}\left(\exp \left(2 n \mu_{V_{a}}+\frac{\left(2 n \sigma_{V_{a}}\right)^{2}}{2}\right)-\left(\exp \left(n \mu_{V_{a}}+\frac{\left(n \sigma_{V_{a}}\right)^{2}}{2}\right)\right)^{2}\right), a \in A
\end{aligned}
$$
that is

The travel time on path can be calculated according to the relationship between link and path,

$$
T_{k}^{w}=\sum_{a \in A} T_{a} \delta_{a, k}^{w}, k \in R_{w}, w \in W
$$

Where $T_{k}^{w}$ is the travel time of path $k$ between the OD pair $w$.

It is assumed that the link travel times are independent of each other. The mean of path travel time $t_{k}^{w}$ and the variance of path travel time $\varepsilon_{T_{k}^{w}}$ can then be expressed as

$$
\begin{aligned}
& t_{k}^{w}=\sum_{a \in A} \delta_{a, k}^{w} E\left(T_{a}\right)=\sum_{a \in A} \delta_{a, k}^{w}\left(t_{a 0}+\frac{\beta t_{a 0}}{\left(c_{a}\right)^{n}} \exp \left(n \mu_{V_{a}}+\frac{\left(n \sigma_{V_{a}}\right)^{2}}{2}\right)\right), k \in R_{w}, w \in W \\
& \varepsilon_{T_{k}^{w}}=\operatorname{var}\left(T_{k}^{w}\right)=\sum_{a \in A} \delta_{a, k}^{w} \operatorname{var}\left(T_{a}\right)=\sum_{a \in A} \delta_{a, k}^{w} \varepsilon_{T_{a}}, k \in R_{w}, w \in W
\end{aligned}
$$

The path travel time $T_{k}^{w}$ can be expressed by summing the corresponding link travel time Ta, Therefore, $T_{k}^{w}$ is approximately follows the normal distribution according to the central limit theorem ${ }^{[12]}$, that is

$$
T_{k}^{w} \sim N\left(t_{k}^{w},\left(\sqrt{\varepsilon_{T_{k}^{w}}}\right)^{2}\right), k \in R_{w}, w \in W
$$

The probability density function of $T_{k}^{w}$ is denoted as $f_{T_{k}^{w}}(x)$

$$
f_{T_{k}^{w}}(x)=\frac{1}{\sqrt{2 \pi} \sigma_{T_{k}^{w}}} \exp \left(-\left(x-t_{k}^{w}\right)^{2} /\left(2\left(\sigma_{T_{k}^{w}}\right)^{2}\right)\right),-\infty<x<\infty
$$

Where $\sigma_{T_{k}^{w}}=\sqrt{\varepsilon_{T_{k}^{w}}}, k \in R_{w}, w \in W$.

Cumulative prospect theory decision system. The decision-making system of cumulative prospect theory mainly includes the value function, decision weight function and the the calculation formula of prospect value ${ }^{[8,9]}$. Travelers' path choice is based on the prospect value of the travel path, and the calculation of the prospect value depends on the value function and the decision 
weight function according to the cumulative prospect theory. The value function is the subjective utility of the actual utility of each path, which is measured by the value of the deviation from the reference point, that is, return or loss. Decision weighting function reflects the subjective probability based on the occurrence of the actual probability of the path utility.

Value function. According to the cumulative prospect theory, the value function can be expressed as:

$$
\varphi_{w}\left(T_{k}^{w}\right)=\left\{\begin{array}{c}
\left(\pi_{w}-T_{k}^{w}\right)^{\alpha}, T_{k}^{w} \leq \pi_{w} \\
-\lambda\left(T_{k}^{w}-\pi_{w}\right)^{\beta}, T_{k}^{w}>\pi_{w}
\end{array}, k \in R_{w}, w \in W\right.
$$

Where $\varphi_{w}\left(T_{k}^{w}\right)$ represents the value function of path $k$ between the OD pair $w$, $\pi_{w}$ expresses the reference point of travel time between the OD pair $w$, when the path travel time is less than $w$, it means return for traveler, otherwise, it means loss. $\alpha$ and $\beta(0<\alpha, \beta \leq 1)$ are the risk sensitivity coefficient, which indicate that the decision maker is risk averse when facing the return and would become risk preference under the loss, and $\alpha$ and $\beta$ also express the marginal decline characteristic of the value function. $\lambda(\lambda \geq 1)$ is the loss aversion coefficient which indicates decision maker is more sensitive to the loss than the same return.

Decision weight function. A typical decision weight function can be shown below:

$$
w(p)=\exp \left(-(-\ln p)^{\gamma}\right)
$$

Where the actual probability and the perceived probability are denoted as $p$ and $w(p)$ respectively, the parameter $\gamma(0<\gamma \leq 1)$ indicates that the decision maker will overestimate the small probability events and underestimate the large probability events.

Prospect value. The prospect value of chosen path can be calculated according to the value function and decision weight function on the condition that the distribution of the travel time of the path has been known, as shown blow

$$
v_{k}^{w}=\int_{t_{k}^{w}}^{\pi_{w}} \frac{d w\left(\psi_{k}^{w}(x)\right)}{d x} \varphi_{w}(x) d x+\int_{\pi_{w}}^{-t_{k}^{w}}-\frac{d w\left(1-\psi_{k}^{w}(x)\right)}{d x} \varphi_{w}(x) d x, \quad k \in R_{w}, w \in W
$$

Where $v_{k}^{w}$ is the prospect value of path $k$ between the OD pair $w \cdot \psi_{k}^{w}(\cdot), t_{k}^{w}$ and $t_{k}^{-w}$ are the cumulative distribution function, lower and upper limit value of the travel time on path $k$ between the OD pair $w$ respectively.

The choice of reference point. The choice of reference point has significant influence on the prospect value of path and the distribution of the network equilibrium flow. Empirical studies has shown that the travelers not only want to save the travel time, but also want to improve the reliability of the trip in the random network environment. Lo H.K. et al proposed the concept of time budget travel (TTB) ${ }^{[12]}$, which was used to describe the reliability of the path choice. Xu H.L. et $\mathrm{al}^{[13]}$ took travel time budget as endogenous reference point when choosing the path. Travel time budget can be expressed as:

$$
\xi_{k}^{w}(\omega)=t_{k}^{w}+\Phi^{-1}(\omega) \sigma_{T_{k}^{w}}, k \in R_{w}, w \in W
$$

Where $\Phi^{-1}($.$) is the inverse function of the standard normal distribution. \omega$ denotes the reliability demand of traveling, which means the travelers are more likely to avoid the traveling risk the with the greater value. $\xi_{k}^{w}(\omega)$ is the budget of traveling time while the value of reliability demand degree is $\omega$.

Then calculate the reference point:

$$
\pi_{w}=\min _{k \in R_{w}}\left\{\xi_{k}^{w}(\omega)\right\}, w \in W
$$

Stochastic user equilibrium model based on CPT. It is difficult for travelers to master the distribution regularities of the travel time in actual traffic environment, which may lead to the estimation deviation of the prospect value of the path. The estimation of prospect value of path can be considered as a random variable, which is determined by both the distribution of the actual 
observation value $v_{k}^{w}$ and the random error term $\zeta_{k}^{w}$, as shown below

$$
V_{k}^{w}=v_{k}^{w}+\zeta_{k}^{w}, k \in R_{w}, w \in W
$$

Where $V_{k}^{w}$ is the estimated prospect value of path $k$ between the OD pair $w$.

Supposing $\zeta_{k}^{w}$ is the gumbel variable that follows the independent and identically distribution and the expected value is 0 , then the choice probability of effective path $k$ between the OD pair $w$, $p_{k}^{w}$, can be formulated using the following Logit form:

$$
p_{k}^{w}=\frac{\exp \left(\theta v_{k}^{w}\right)}{\sum_{r \in R_{w}} \exp \left(\theta v_{r}^{w}\right)}, k \in R_{w}, w \in W
$$

Where $\theta$ is the dispersion parameter, which is inversely proportional to the standard error of the distribution of the perceived cost of travel route.

Assuming the perceived prospect value of the path is the basis of the route choice. All the travelers try to find the path with the maximum prospect value in the process of choosing path, the network will reach a random user equilibrium state based on the cumulative prospect theory when the travelers can not improve the prospect value through the unilateral change of the path. At the same time, the value of all the paths chosen in the same OD are equal, and are greater than or equal to the prospect value of the path that have not been chosen. According to the stochastic user equilibrium theory, the stochastic user equilibrium condition based on the cumulative prospect theory can be expressed as following:

$$
f_{k}^{w}=q_{w} p_{k}^{w}, k \in R_{w}, w \in W
$$
That is

The equilibrium conditions of the formula (35) can be described by the variational inequality.

$$
\sum_{w \in W} \sum_{k \in R_{w}}\left(s_{w}+\frac{1}{\theta} \ln \frac{f_{k}^{w^{*}}}{q_{w}}-v_{k}^{w}\right)\left(f_{k}^{w}-f_{k}^{w^{*}}\right) \geq 0, \forall f_{k}^{w} \in \Omega
$$

Where $f_{k}^{w^{*}}$ is the solution of the variational inequality, $\Omega$ denotes the feasible set determined by formula 4 to $6 . s_{w}$ is the expected maximum estimated prospect value between the OD pair $w$, also called satisfactory function, which can be calculated by the following formula ${ }^{[14]}$

$$
s_{w}=E\left(\max _{k \in R_{w}}\left\{V_{k}^{w}\right\}\right)=\frac{1}{\theta} \ln \left(\sum_{k \in R_{w}} \exp \left(\theta v_{k}^{w}\right)\right), w \in W
$$

$f$ denotes the column vector composed of $\left\{f_{k}^{w}\right\}, v(f)$ is the column vector composed of

$\left\{s_{w}+\frac{1}{\theta} \ln \frac{f_{k}^{w}}{q_{w}}-v_{k}^{w}\right\}$. Since $\boldsymbol{v}(\boldsymbol{f})$ is a continuous function about $\boldsymbol{f}$, and the feasible set is bounded closed convex set, there are at least one solution of variational inequality represented by formula (36) according to the variational inequality theorem ${ }^{[15]}$. However, the uniqueness of the solution can't be guaranteed due to the uncertainty of monotony of $v(f)$.

The method of successive average (MSA) algorithm can be used to solve the variational inequality, and the algorithm steps are omitted because of the limited space.

\section{The model for network vulnerability evaluation based on CPT}

The research has shown that the path choice behavior of travelers presents the characteristic of limited rationality in the random network environment, which is accordant with the decision framework of CPT. According to the conclusion of research ${ }^{[7]}$, the neglect of the travelers' behavior response in the vulnerability evaluation of road network will lead to wrong judgment of vulnerability, and even wrong decision. The identification of critical links is one of the most critical issues in the vulnerability evaluation of road network, and the failure of those links would have the 
most serious impacts on the whole network. The definition of validity index of road network based on the CPT is proposed in this paper, and the vulnerability is measured by the change of road network availability, thus the critical links are identified. The validity index is defined as below:

$$
E(G)=\sum_{w \in W} q_{w} s_{w} / \sum_{w \in W} q_{w}
$$

Where $E(G)$ indicates the validity of the road network $G$.

The consequence of the failure of link a in the network is quantified by the vulnerability index, denoted as $V U L_{a}$. It can be calculated by

$$
V U L_{a}=\frac{E_{0}(G)-E_{a}(G)}{E_{0}(G)}, a \in A
$$

Where $E_{0}(G)$ and $E_{a}(G)$ respectively are the network efficiency of network $G$ under the normal condition and after the closure of link a. Obviously, $V U L_{a}$ reflects the change of road network efficiency caused by the failure of link $a$.

The steps of the vulnerability evaluation scheme of road network based on the above analysis are given as flowing:

Step 1: Calculate the network efficiency of network $G \quad E_{0}(G)$ under the normal condition. The equilibrium path flow and the expected maximum prospect value $s_{w}$ are calculated through the MSA algorithm, then calculate the $E_{0}(G)$.

Step 2: Calculate the network efficiency of network $G E_{a}(G)$ after the failure of link. Each link is iteratively removed from the network and the consequences of its closure are measured in terms of the reduced network performance.

Step 3: Calculate the road network vulnerability $V U L_{a}$. The road network vulnerability index after the failure of each link is calculated in turn which is on the basis of $E_{0}(G)$ and $E_{a}(G)$ according to the formula (39) .

Step 4: Identify the critical links. Let rank $_{a}$ be the vulnerable ranking of link a closure, determine the $r a n k_{a}$ values by sorting the $V U L_{a}$ values in a descendent order, then select the first $N$ links with the minimum values of the $\operatorname{rank}_{a}$ as the critical links, where $N$ is the number of critical links set in advance.

Where the steps of MSA algorithm are as following:

Step 1: Initialization. Set $\tau=0.01, n=1$, and initialize the path flow $\boldsymbol{f}^{(n)}=\left\{f_{k}^{w(n)}\right\}_{k \in R_{w}, w \in W}$, where $\tau$ is the Error parameter and $n$ is the iteration number.

Step 2: Calculate the distribution of path traveling time and the vector of travel prospect value based on the current path flow $\boldsymbol{f}^{(n)}$, that is $\boldsymbol{f}^{(n)}=\left\{f_{k}^{w(n)}\right\}_{k \in R_{w}, w \in W}$.

Step 3: Search the iterative direction $\boldsymbol{g}^{(n)}$, set $\boldsymbol{g}^{(n)}=\boldsymbol{f}^{(n)^{\prime}}-\boldsymbol{f}^{(n)}$. Where $f_{k}^{w(n)^{\prime}}=q_{w} \frac{\exp \left(\theta v_{k}^{w(n)}\right)}{\sum_{r \in R_{w}} \exp \left(\theta v_{r}^{w(n)}\right)}$ $k \in R_{w}, w \in W$.

Step 4: Update. Set $\boldsymbol{f}^{(n+1)}=\boldsymbol{f}^{(n)}+\frac{1}{n} \boldsymbol{g}^{(n)}$.

Step 5: Check the convergence. If $\max _{w \in W}\left|\frac{s_{w}^{(n)}-s_{w}^{(n-1)}}{s_{w}^{(n)}}\right|<\tau$, then stop iteration, otherwise, set $n=n+1$, turn to step 2 .

\section{Numerical examples}

The Nguyen and Dupuis network is taken as the test network, which is shown as Figure 1. The network consists of 4 OD pairs, 13 nodes, 19 sections and 25 paths. The number of link is the 
number identified in the links, and there are some assumptions about performance parameters of link, the travel time of free flow is 12 minute except link 4, link13 and link36 of which travel time is $24,24,36$ minute respectively, the design capacity of link is assumed to be 2500 vehicle per hour except link 13 and link 18 of which the design capacity is 1500 vehicle per hour.

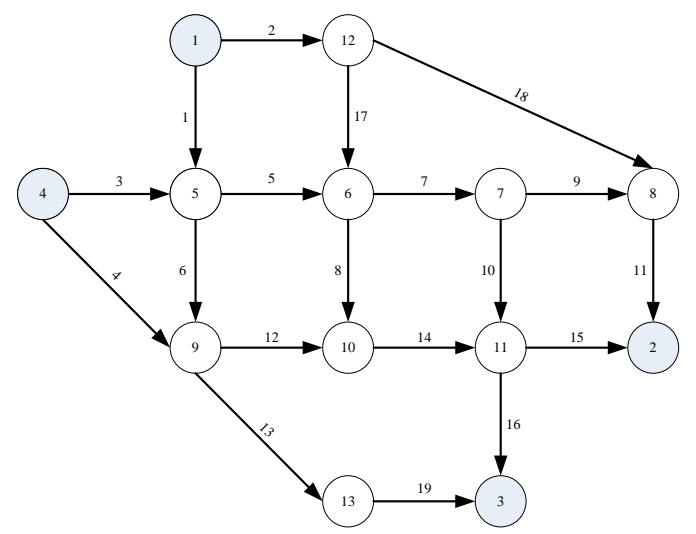

Fig. 1 Nguyen and Dupuis Network

Without loss of generality and rationality, the test parameters are set as follows: $V M R_{w}=0.4, w \in W ; \beta_{0}=0.15, n=4 ; \omega=80 \% ; \alpha=\beta=0.88, \lambda=2.25, \gamma=0.65 ; \theta=0.3 ; N=10$, the value of lower limit of reference point is the travel time of the free flow, namely $t_{-k}^{w}=\sum_{a \in A} \delta_{a, k}^{w} t_{a 0}$, the upper limit ${ }_{t}^{-w}$ is the budget of travel time when the reliability reaches 99.999\%. The mean of OD demand is expressed as follows for ease of comparison:

$q_{w}=z q_{w}^{0}, w \in W$

Where $z$ is the demand multiplier, and $0 \leq \mathrm{z} \leq 1 . q_{w}^{0}$ is the benchmark demand, set $q_{12}=2000 \mathrm{veh} \cdot \mathrm{h}^{-1}, \quad q_{13}=1500 \mathrm{veh} \cdot \mathrm{h}^{-1}, q_{42}=1500 \mathrm{veh} \cdot \mathrm{h}^{-1}, \quad q_{43}=1500 \mathrm{veh} \cdot \mathrm{h}^{-1}$. Set $z=1$ without special instruction.

The results of road network vulnerability evaluation when the demand coefficient is $z=1$ are given in Table 1, only the first 10 links with highest vulnerability are listed due to the limitation of space. It is obvious that the failure of any one of these links will lead to the decline of validity of road network at least $20 \%$. And the failure of link 14 has the most significant impact on the validity of road network, of which the vulnerability is up to $48.39 \%$, the validity of road network dropped by nearly half.

Table.1 The Results of Network Vulnerability Evaluation

\begin{tabular}{c|c|c|c|c|c|c|c}
\hline rank $_{a}$ & $E_{a}(G)$ & $V U L_{a}$ & $\begin{array}{c}\text { Failure } \\
\text { link }\end{array}$ & rank $_{a}$ & $E_{a}(G)$ & $V U L_{a}$ & $\begin{array}{c}\text { Failure } \\
\text { link }\end{array}$ \\
\hline 1 & 2.190 & $48.39 \%$ & 14 & 6 & 3.230 & $23.87 \%$ & 1 \\
\hline 2 & 2.459 & $42.05 \%$ & 7 & 7 & 3.245 & $23.53 \%$ & 16 \\
\hline 3 & 2.705 & $36.24 \%$ & 15 & 8 & 3.323 & $21.69 \%$ & 6 \\
\hline 4 & 2.852 & $32.79 \%$ & 3 & 9 & 3.338 & $21.32 \%$ & 2 \\
\hline 5 & 2.995 & $29.42 \%$ & 5 & 10 & 3.355 & $20.92 \%$ & 17 \\
\hline
\end{tabular}

The influence of factors on the vulnerability of road network, such as the congestion level, fluctuation degree of demand, reliability requirement and traveler route choice behavior is listed in Table 2 to Table 5 respectively where only 10 links are listed. The effect of parameters, such as $\alpha$, $\beta, \gamma$ on the road network vulnerability is not significant and the tables are not listed here. With the changing of the parameters, the variation is not obvious no matter what the road network vulnerability degree or the key degree of links.

It can be seen clearly from the Table 2 that the vulnerability of road network presents a rising trend with the increase of the congestion degree, namely the failure of link will result more 
significant impact on road network vulnerability. In addition, it has shown that the critical links are different with the change of road congestion degree from the data in Table 2. Table 3 shows that the fluctuation degree of OD demand has certain impact on the road network vulnerability, the specific performance is that the road network vulnerability will be reduced with the increase of the variance-mean ratio of the demand, and the key degree of some links will be changed. The reason for this phenomenon is that the value of the reference point will increase when the variance-mean ratio increases, which means the increasing probability of revenue and the validity of road network as well as the decreasing of vulnerability.

Table.2 The Effects of Congestion level on Network Vulnerability

\begin{tabular}{|c|c|c|c|c|c|c|c|c|c|c|c|c|c|}
\hline \multirow{2}{*}{ Link } & \multicolumn{2}{|c|}{$z=0.1$} & \multicolumn{2}{|c|}{$z=0.5$} & \multicolumn{2}{|c|}{$z=0.9$} & \multirow{2}{*}{ ink } & \multicolumn{2}{|c|}{$z=0.1$} & \multicolumn{2}{|c|}{$z=0.5$} & \multicolumn{2}{|c|}{$z=0.9$} \\
\hline & $V U L_{a}$ & $\operatorname{rank}_{c}$ & $V U L_{a}$ & $\operatorname{rank}_{a}$ & $V U L_{a}$ & $\operatorname{rank}_{a}$ & & $V U L_{a}$ & rank $_{a}$ & $V U L_{a}$ & rank $_{c}$ & $V U L_{a}$ & $\operatorname{rank}_{a}$ \\
\hline 1 & $27.45 \%$ & 6 & $27.44 \%$ & 7 & $25.09 \%$ & 7 & 6 & $14.63 \%$ & 12 & $17.47 \%$ & 12 & $21.54 \%$ & 9 \\
\hline 2 & $18.05 \%$ & 8 & $19.67 \%$ & 9 & $21.43 \%$ & 10 & 7 & $26.71 \%$ & 7 & $38.97 \%$ & 3 & $43.69 \%$ & 2 \\
\hline 3 & $31.79 \%$ & 4 & $33.06 \%$ & 4 & $32.77 \%$ & 4 & 8 & $15.39 \%$ & 11 & $15.71 \%$ & 13 & $15.36 \%$ & 14 \\
\hline 4 & $6.70 \%$ & 16 & $7.57 \%$ & 16 & $9.31 \%$ & 16 & 9 & $8.25 \%$ & 15 & $11.92 \%$ & 15 & $16.45 \%$ & 13 \\
\hline 5 & $30.12 \%$ & 5 & $32.17 \%$ & 6 & $31.21 \%$ & 5 & 10 & $15.42 \%$ & 10 & $20.64 \%$ & 8 & $21.13 \%$ & 11 \\
\hline
\end{tabular}

Table.3The Effects of Demand Variation Degree on Network Vulnerability

\begin{tabular}{|c|c|c|c|c|c|c|c|c|c|c|c|c|c|}
\hline \multirow{2}{*}{ Link } & \multicolumn{2}{|c|}{$V M R_{w}=0.10$} & \multicolumn{2}{|c|}{$V M R_{w}=0.50$} & \multicolumn{2}{|c|}{$V M R_{w}=0.90$} & \multirow[b]{2}{*}{ Link } & \multicolumn{2}{|c|}{$V M R_{w}=0.10$} & \multicolumn{4}{|c|}{\begin{tabular}{l|l|}
$V M R_{w}=0.50$ & $V M R_{w}=0.90$
\end{tabular}} \\
\hline & $V U L_{a}$ & $\operatorname{rank}_{c}$ & $V U L_{a}$ & $\operatorname{rank}_{a}$ & $V U L_{a}$ & rank & & $V U L_{a}$ & $\operatorname{rank}_{\mathrm{c}}$ & $V U L_{a}$ & $\operatorname{rank}_{a}$ & $V U L_{a}$ & $\overline{\operatorname{rank}_{a}}$ \\
\hline 1 & $24.95 \%$ & 7 & $23.63 \%$ & 6 & $22.82 \%$ & $\overline{6}$ & 6 & $21.91 \%$ & $\overline{9}$ & $21.65 \%$ & $\overline{8}$ & $21.47 \%$ & 7 \\
\hline 2 & $23.82 \%$ & 8 & $20.79 \%$ & 10 & $19.19 \%$ & 11 & 7 & $43.51 \%$ & 2 & $41.74 \%$ & 2 & $40.74 \%$ & 2 \\
\hline 3 & $33.22 \%$ & 4 & $32.71 \%$ & 4 & $32.43 \%$ & 4 & 8 & $14.87 \%$ & 14 & $14.76 \%$ & 14 & $14.61 \%$ & 14 \\
\hline 4 & $9.10 \%$ & 16 & $8.86 \%$ & 15 & $8.64 \%$ & $\overline{15}$ & 9 & $\%$ & 13 & $15.82 \%$ & 13 & $15.54 \%$ & 13 \\
\hline 5 & $30.24 \%$ & 5 & $29.24 \%$ & 5 & $28.66 \%$ & 5 & 10 & $20.01 \%$ & 11 & $19.57 \%$ & 11 & $19.21 \%$ & 10 \\
\hline
\end{tabular}

Table 4 shows that the road network vulnerability will decrease along with the increasing of reliability demand. The reason is similar to the effects resulted from the demand fluctuation, that is the value of the reference point will increase when the reliability demand increases, which means the increasing probability of revenue and the road network validity as well as the decreasing of impact of link closure on vulnerability.

Table.4 The Effects of Probability of Reliability on Network Vulnerability Evaluation

\begin{tabular}{|c|c|c|c|c|c|c|c|c|c|c|c|c|c|}
\hline \multirow{2}{*}{ Link } & \multicolumn{2}{|c|}{$\omega=0.50$} & \multicolumn{2}{|c|}{$\omega=0.70$} & \multicolumn{2}{|c|}{$\omega=0.90$} & & \multicolumn{2}{|c|}{$\omega=0.50$} & \multicolumn{2}{|c|}{$\omega=0.70$} & \multicolumn{2}{|c|}{$\omega=0.90$} \\
\hline & $V U L_{a}$ & rank & $V U L_{a}$ & rank & $V U L_{a}$ & $\operatorname{rank}_{c}$ & & $V U L_{a}$ & $\operatorname{rank}_{a}$ & $V U L_{a}$ & $\operatorname{rank}_{a}$ & $V U L_{a}$ & $\operatorname{rank}_{a}$ \\
\hline 1 & $28.53 \%$ & 7 & $25.52 \%$ & 7 & $21.75 \%$ & 6 & 6 & $22.77 \%$ & 9 & $22.05 \%$ & 9 & $21.23 \%$ & 7 \\
\hline 2 & $27.91 \%$ & 8 & $23.70 \%$ & 8 & $18.20 \%$ & 10 & 7 & $47.89 \%$ & 2 & $44.13 \%$ & 2 & $39.38 \%$ & 2 \\
\hline 3 & $34.92 \%$ & 5 & $33.54 \%$ & 4 & $31.89 \%$ & 4 & 8 & $15.17 \%$ & 14 & $14.92 \%$ & 14 & $14.60 \%$ & 14 \\
\hline 4 & $9.80 \%$ & 16 & $9.22 \%$ & 16 & $8.50 \%$ & 15 & 9 & $16.88 \%$ & 13 & $16.24 \%$ & 13 & $15.41 \%$ & 13 \\
\hline 5 & $32.12 \%$ & 6 & $30.38 \%$ & 5 & $28.17 \%$ & 5 & 10 & $20.94 \%$ & 11 & $20.11 \%$ & 11 & $19.06 \%$ & 9 \\
\hline
\end{tabular}

Table.5 The Effects of Travelers' Route Choice Behavior on Network Vulnerability Evaluation

\begin{tabular}{|c|c|c|c|c|c|c|c|c|c|}
\hline \multirow{2}{*}{ Link } & \multicolumn{2}{|c|}{$\begin{array}{c}\text { Bounded } \\
\text { ionality hypothesis }\end{array}$} & \multicolumn{2}{|c|}{$\begin{array}{c}\text { Complete Rational } \\
\text { hypothesis }\end{array}$} & \multirow[t]{2}{*}{ Link } & \multicolumn{4}{|c|}{$\begin{array}{c}\begin{array}{c}\text { Bounded rationality } \\
\text { hypothesis }\end{array} \\
\begin{array}{c}\text { hyplete rational } \\
\text { hypothesis }\end{array} \\
\end{array}$} \\
\hline & $V U L_{a}$ & rank $_{a}$ & $V U L_{a}$ & $\operatorname{rank}_{a}$ & & $V U L_{a}$ & $\operatorname{rank}_{a}$ & $V U L_{a}$ & $\overline{\operatorname{rank}_{a}}$ \\
\hline 1 & $23.87 \%$ & 6 & $9.30 \%$ & 5 & 6 & $21.69 \%$ & 8 & $3.40 \%$ & 13 \\
\hline 2 & $21.32 \%$ & 9 & $11.60 \%$ & 2 & 7 & $42.05 \%$ & 2 & $8.95 \%$ & 7 \\
\hline 3 & $32.79 \%$ & 4 & $5.59 \%$ & 9 & 8 & $14.78 \%$ & 14 & $1.43 \%$ & 19 \\
\hline 4 & $8.91 \%$ & 15 & $2.48 \%$ & 16 & 9 & $15.88 \%$ & 13 & $2.32 \%$ & 17 \\
\hline 5 & $29.42 \%$ & 5 & $5.64 \%$ & 8 & 10 & $19.66 \%$ & 11 & $2.78 \%$ & 15 \\
\hline
\end{tabular}


Table 5 shows the of the vulnerability evaluation of the road network in different assumptions of travelers' path choice. The special explanation is elucidated in this paper, the traditional stochastic user equilibrium model is used to carry on the flow assignment assuming that the travel time budget is the basis for the path choice on the condition that the travels are bounded rationality. Moreover, because the travel time is considered as the side effect, the degree of satisfaction can be calculated as:

$$
s_{w}=-\frac{1}{\theta} \ln \left(\sum_{k \in R_{w}} \exp \left(-\theta \xi_{k}^{w}(\omega)\right)\right), w \in W
$$

The travelers' route choice behavior will have a significant impact on the road network vulnerability, which can be seen from the data in Table 5. The road network vulnerability and the key degree of links are obviously different because of the different assumptions of path choice behavior. The wrong assumption of path choice behavior will lead to the wrong identification of road network vulnerability and critical links.

As shown in Figure 2, the change of road network vulnerability presents nonlinear state in the impact of the combination of demand fluctuation, congestion level and reliability. The whole trend is the road network vulnerability will increase with the increasing of congestion degree, but the road network vulnerability will decrease with the increasing of demand fluctuation and reliability.

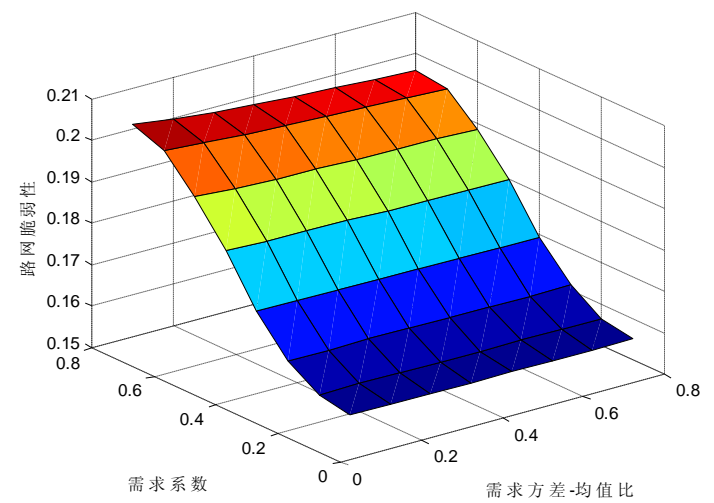

(a) The impact of the combination of demand fluctuation and congestion level

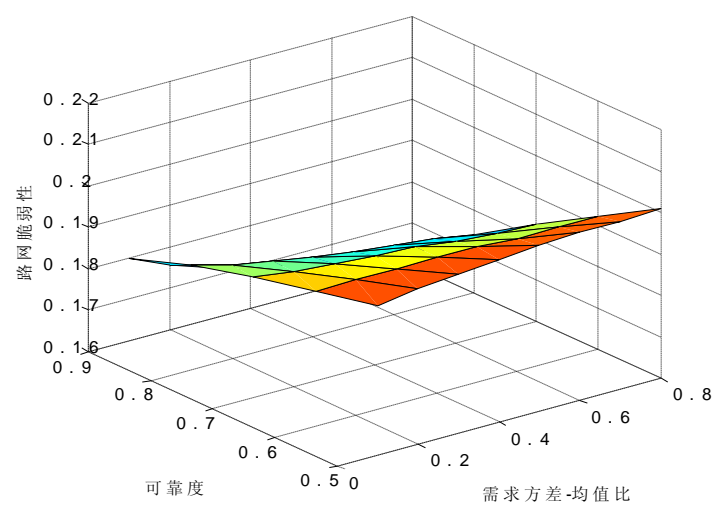

(b) The impact of the combination of demand fluctuation and reliability

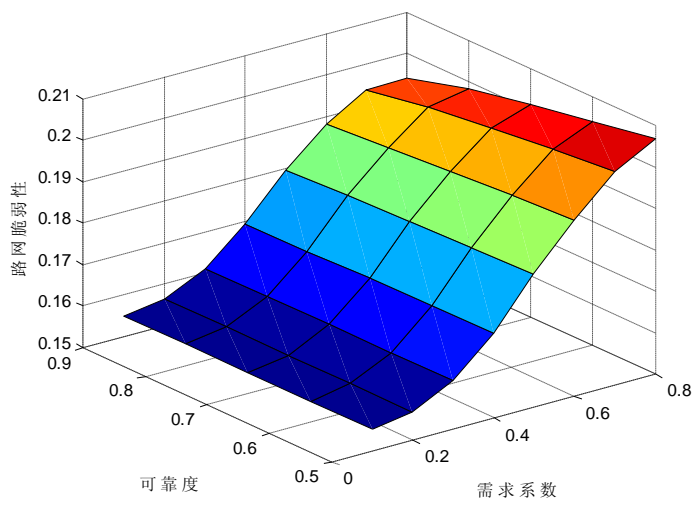

(c) The impact of the combination of demand levels and reliability

Fig.2 The Network Vulnerability under mutual Combinations of Various Demand Levels, Congestion degrees and Reliability Levels (after Link 12 Closure) 


\section{Conclusion}

In this paper, a model for network vulnerability evaluation based on cumulative prospect theory is proposed, and the effects of travelers' route choice behavior with bounded rationality are explicitly taken into account. And the stochastic user equilibrium model based on the cumulative prospect theory is established. The definition of network efficiency index based on cumulative prospect value is proposed, and the vulnerability is measured by the change of network efficiency index, and then the critical links that the failure of those links would have significant impacts on the whole network can be identified. Nguyen and Dupuis network is used as the test network to verify the influence of the factors on the vulnerability. The result has shown that travelers' route choice behavior, congestion degree of network and the demand fluctuation have an important impact on the vulnerability evaluation of road network, and the ignorance of these factors may lead to wrong judgment of the road network vulnerability and false identification of critical links.

The proposed model only considers the scenarios of single link closure, and the impact of multiple link closures on the vulnerability should be taken into consideration in the next study. And it is not reasonable to assume that all the travelers have the same risk, further studies are also required to study the heterogeneity of traveler for confirming the different types of reference points, which benefits to model travelers' behavior response to the link closure.

\section{Acknowledgements}

This work was financially supported by The Soft Science Plan Project of Sichuan Province (2015ZR0155) and National Natural Science Foundation of China (51278429).

\section{Reference}

[1] Yin Hongying, Xu Liqun. Vulnerability Assessment of Transportation Road Networks [J]. Journal of Transportation Systems Engineering and Information Technology, 2010, 10(3): 7-13.

[2] Berdica K. An introduction to road vulnerability: what has been done, is done and should be done [J]. Transport Policy, 2002, 9(2): 117-127.

[3] Jenelius E, Petersen T, Mattsson L-G. Importance and exposure in road network vulnerability analysis [J]. Transportation Research Part A, 2006, 40(7): 537-560.

[4] Taylor M A P, Sekhar S V C, D'Este G M. Application of accessibility based methods for vulnerability analysis of strategic road networks [J]. Networks and Spatial Economics, 2006, 6(3): 267-291.

[5] Chen A, Yang C, Kongsomsaksakul S, et al. Network-based accessibility measures for vulnerability analysis of degradable transportation networks [J]. Networks and Spatial Economics, 2007, 7(3): 241-256.

[6] Yang Luping, Qian Dalin. Vulnerability Analysis of Road Networks [J]. Journal of Transportation Systems Engineering and Information Technology, 2012, (1): 5-10.

[7] Chen B Y, Lam W H K, Sumalee A, et al. Vulnerability analysis for large-scale and congested road networks with demand uncertainty [J]. Transportation Research Part A, 2012, 46(3): 501-516.

[8] Tversky A, Kahneman D. Advances in prospect theory: Cumulative representation of uncertainty [J]. Journal of Risk and Uncertainty, 1992, 5(4): 297-323.

[9] Avineri E. The effect of reference point on stochastic network equilibrium [J]. Transportation Science, 2006, 40(4): 409-420.

[10]Chen A., Zhou Z. The a-reliable mean-excess traffic equilibrium model with stochastic travel times. Transportation Research Part B, 2010, 44(4): 493-513.

[11]Shao H., Lam W H.K., Tam M.L. A reliability-based stochastic traffic assignment model for network with multiple user classes under uncertainty in demand. Network and Spatial 
Economics. 2006, 6(3): 173-204.

[12]Lo H.K., Luo X.W., Siu B W.Y. Degradable transport network: Travel time budget of travelers with heterogeneous risk aversion [J]. Transportation Research Part B, 2006, 40(9): 792-806.

[13] Xu H.L., Lou Y.Y., Yin Y.F. A prospect-based user equilibrium model with endogenous reference points and its application in congestion pricing. Transportation Research Part B, 2011, 45(2): 311-328.

[14]Huang H.J. Urban Transportation Network Equilibrium Analysis: Theory and Practice. Beijing: China Communications Press.1994.

[15]Facchinei F, Pang J.S. Finite dimensional Variational Inequalities and Complex entarity Problems. Berlin Heidelberg, New York: Springer. 2003. 